\title{
Distal displacement of maxillary sinus anterior wall versus conventional sinus lift with lateral access: a 3-year retrospective Computerized Tomography study
}

\section{Roberto Crespi}

Universita degli Studi Guglielmo Marconi

\section{Paolo Toti}

Universita degli Studi Guglielmo Marconi

\section{Ugo Covani}

tuscan stomatologic institute

\section{Carlo Bruno Brevi}

Universita degli Studi di Pisa

\section{Luigi Rubino}

Universita degli Studi Guglielmo Marconi

\section{Camilla Preda}

Universita degli Studi Guglielmo Marconi

\section{Francesca Esposito}

Universita degli Studi Guglielmo Marconi

Giovanni-Battista Menchini-Fabris ( $\nabla$ gb.menchinifabris@gmail.com )

Universita degli Studi Guglielmo Marconi https://orcid.org/0000-0002-7646-8967

Research article

Keywords: Dental implant, maxillary sinus, bone augmentation,CT imaging, infracture approach

Posted Date: March 17th, 2020

DOl: https://doi.org/10.21203/rs.3.rs-17479/v1

License: (c) (i) This work is licensed under a Creative Commons Attribution 4.0 International License. Read Full License

Version of Record: A version of this preprint was published at International Journal of Environmental Research and Public Health on October 1st, 2020. See the published version at 
https://doi.org/10.3390/ijerph17197199. 


\section{TITLE PAGE}

\section{TITLE}

Distal displacement of maxillary sinus anterior wall versus conventional sinus lift with lateral access: a 3-year retrospective Computerized Tomography study

\section{AUTHORS}

Crespi Roberto, MD, MSc in Dentistry, Professor $\$$

Toti Paolo, BSc, DDS, Visiting Professor $\dagger$

Covani Ugo, MD, DDS, Professor ^

Brevi Bruno, MD, Acting Director *

Rubino Luigi, MD, DDS, Professor \#

Preda Camilla ,RDH, Dental School Student \%

Francesca Esposito, RDH, Dental School Student §

Menchini-Fabris Giovanni-Battista, DDS, Professor ${ }^{\circ}$

\section{DEPARTMENT AND INSTITUTIONS}

¥ Study Center for Regenerative Research, University "G. Marconi”, Rome Italy and Tuscan Dental Institute, Versilia Hospital - Camaiore (Italy)

$\dagger$ Study Center for Regenerative Research, University "G. Marconi", Rome Italy and Tuscan Dental Institute, Versilia Hospital - Camaiore (Italy)

^ Department of Surgical, Medical, Molecular and Critical Area Pathology, University of Pisa and Tuscan Dental Institute, Versilia Hospital - Camaiore (Italy)

* Department of Maxillo-Facial Surgery (Acting Director: Dr. Bruno Brevi), Hospital and University of Pisa - Pisa (Italy)

\# Department of Multidisciplinary Regenerative Research, "Guglielmo Marconi University”, Rome, Italy AND Tuscan Dental Institute, Versilia Hospital - Camaiore (Italy)

\% Department of Multidisciplinary Regenerative Research, "Guglielmo Marconi University”, Rome, Italy

$\S$ Department of Multidisciplinary Regenerative Research, “Guglielmo Marconi University”, Rome, Italy

○ Study Center for Regenerative Research, University "G. Marconi", Rome Italy and Tuscan Dental Institute, Versilia Hospital - Camaiore (Italy) 


\section{CORRESPONDING AUTHOR}

Department of Multidisciplinary Regenerative Research, "Guglielmo Marconi University”, Via Vittoria Colonna, 11, 00193 Rome, Italy

Private Practice

San Rossore Dental Unit

Viale delle Cascine 152 San Rossore, 56122 Pisa PI

+393397157007 / +39050578815

e-mail: gb.menchinifabris@gmail.com

\section{RUNNING HEAD}

Displacement of maxillary sinus anterior wall 


\section{ABSTRACT}

\section{Background}

To compare a sinus augmentation procedure with a distal displacement of the anterior wall to a standard sinus lifting and grafting with a lateral window approach.

\section{Methods}

In the displacement group, a sinus surgical fracture results in the distal displacement of the anterior wall by means of an electromagnetic device. In the filling group, a sinus lifting with lateral access and grafting with particulate bone was performed. Bone volume beneath the maxillary sinus was investigated with Computerized Tomography after data superimposition. Clinical and radiological outcomes over 3 years had been evaluated.

\section{Results}

Forty-three dental implants were enrolled. The surgery of both groups significantly increased the bone volume in the VOIs (p-value $\leq 0.0017$ ) respectively for displacement group from $1.17 \pm 0.34 \mathrm{cc}$ to $1.53 \pm 0.39 \mathrm{cc}$ with a final bone volume gain of $+0.36 \pm 0.17 \mathrm{cc}$ and for filling group from $1.24 \pm 0.41 \mathrm{cc}$ to $1.94 \pm 0.68 \mathrm{cc}$ with a bone augmentation of $0.71 \pm 0.31 \mathrm{cc}$. Two implants early failed in the filling group, attesting the 3-year survival rate to $92.6 \%(\mathrm{CI} 95 \%$ : $82.7 \%-100 \%)$. Marginal bone loss at the distal aspect was $1.66 \pm 0.72 \mathrm{~mm}$ and $1.25 \pm 0.78 \mathrm{~mm}$ for displacement and filling group, respectively ( $\mathrm{p}$-value $=0.0497)$.

\section{Conclusions}

The study showed an effective bone gain around dental implants at 3-year of survey both for sinus augmented by backward displacement of the anterior wall $(+34 \%)$ and by sinus lifting with a lateral window approach $(+57 \%)$.

\section{KEYWORDS}

Dental implant; maxillary sinus; bone augmentation; CT imaging; infracture approach 


\section{BACKGROUND}

Implant placement in the posterior maxilla is particularly challenging when compared to other areas since iatrogenic sinus membrane rupture is a commonly encountered complication, especially when the selected implant length is more than the available bone height. This has been identified as a well-documented cause of implant failure in the posterior upper jaw [1].

Since standard length-implants cannot be placed in cases of severe bone deficiencies, bone augmentation procedure may be used to increase the volume of the residual bone. Pneumatization of the maxillary sinus may be observed in the case of resorption of the internal aspect of the sinus walls.

Boyne and James suggested a lateral approach to the maxillary sinus floor [2]. A bone window was opened through the lateral wall; then, autogenous bone marrow harvested from the iliac crest was poured into the sinus cavity. When there was enough bone for primary stability implants were placed simultaneously. On the other hand, they were placed at a later stage when graft healing was achieved. In case the height of the residual bone crest is less than 4 mm [3,4], a twostage procedure may be appropriate. Several studies evaluated the clinical and radiological outcomes in patients who underwent trans-crestal sinus floor elevation (TSFE) [5-7] varying in residual alveolar bone height and use of bone substitute materials $[8,9]$.

As regards alleged alternative rehabilitations without need for bone augmentation, short implants (less than $6 \mathrm{~mm}$ ) could be successfully loaded in maxillary bone with a residual height from 4 to $6 \mathrm{~mm}$, but their long-term prognosis was still now questionable [10].

\section{METHODS}

\section{Aim}

The aim of the present study was to compare bone remodeling at a 3-year follow-up between a distal displacement of the anterior wall of the maxillary sinus with a standard sinus lifting and grafting surgery with a lateral window approach.

\section{Patient selection}

A set of patients among consecutive subjects treated at the Tuscan Stomatologic Institute between February 2012 and April 2015 were retrospectively enrolled for this study. Such patients had been followed up till 3 years after surgery (2012-2017) at the Complex Operating Unit of Maxillo-Facial Surgery of the University of Pisa, Clinical and radiological information was collected. Written informed consent was obtained from all subjects included, and approval 
for this retrospective analysis was obtained from the Ethical Committee of the University of Pisa, Pisa, Italy (Ethical Approval Form 2626/2008 Protocol Number 58183)

Inclusion criteria:

- sinus lifting with either backward displacement of the anterior wall or with lateral access and grafting with particulate bovine graft;

- dental implants placed in the augmented sinus very close to the native anterior wall (premolar area);

- pre-operative and post-operative maxillary Computerized Tomographic scans.

Patients were excluded if any of the following information was in their medical record:

- no loaded implant during the 3-year follow-up

- lack of post-operative radiographic 3-dimensional (3D) data up to 3-year after augmentation;

- preoperative bone thickness between the sinus floor and the edentulous crest less than $3 \mathrm{~mm}$;

- patients without chronic systemic diseases.

- excessive smoking habits (>10 cigarettes a day);

- alcohol or drug abuse;

- patients unwilling or unable to cooperate in maintaining oral hygiene and to follow the medical prescriptions.

All the surgical interventions were performed by a single surgeon; moreover, a single prosthodontist was responsible for providing all prosthetic treatments. This study followed the Declaration of Helsinki on medical protocol and ethics, and the Regional Ethical Review Board of the University of Pisa approved the present retrospective data analysis ().

\section{Surgical procedures}

Proper premedication with antibiotics was given within one hour before surgery, then it was administered for 6 days (amoxicillin or clindamycin - if allergic to penicillin).

In the sinus wall displacement group (displacement), after treatment with local anesthetic $\dagger \dagger$, a partial-thickness flap was raised to preserve the periosteum. The present management of soft tissues (mainly the intactness of the periosteum) is crucial to maintain the integrity of the blood supply and to promote the healing by secondary intention of tissues surrounding implant site [11].

The primary incision was beveled and slightly palatal to vestibularly displace the keratinized residual tissue. Then, preservation of the papillae was accomplished by making releasing incisions few millimeters from the residual teeth.

A vertical fissure was opened within and through the residual alveolar bone with a blade mounted on an electromagnetic device $+1[12,13]$. Such a device was drawn along the crest of the ridge, through the periosteum, cortex and spongiosa, and towards the floor and anterior wall of the maxillary sinus, where the prosthetic implant palatal 
emergency was planned. Rounded tips were used to displace periosteum-free hard tissue mass within the sinus in the posterior-palatal direction along the side of pristine residual bone volume. This procedure allows us to distally push the anterior wall of the sinus following a parallel direction to the palatal vault.

The result was the creation of a new space between the two lateral walls and the mesio-distal as well.

The implant site was created, both distally against the preexisting lateral walls and apically, moving up and compressing with a progressive increasing diameter of bone expanders. Re-orientation towards the ideal (verticalized) prosthetic axis was then obtained extracting gradually the instruments by forcing the tips of the instruments during removal. The final cavity should remain under-dimensioned in both height and width so that the final plunge is produced by the implant itself, which will be stabilized in the native bone available under the floor of the sinus.

External-hexagon rough/machined osseointegrated dental implants $\S \S$ were placed (Figure 1). The flaps were firmly sutured to the mucosa and the periosteum with holding sutures stabilizing the collagen material ** to control the bleeding and to ensure the blood clot stability. After 90 days of healing, implants were loaded.

In the group in which sinus was lifted with particulate bovine bone graft using the lateral access technique (filling), after treatment with local anesthetic $\dagger \dagger$, a lateral wall was fenestrated and an inferior horizontal osteotomy line was positioned, beveled at the sinus floor level; anterior and posterior vertical osteotomies were performed 5 millimeters outside of the borders of the location in which dental implant had to be placed. Using a sinus membrane elevator, the sinus membrane was gently separated from the sinus floor and the lateral wall was removed. Implants were placed according to the manufacturer's protocol. The sinus cavity was grafted using $100 \%$ particulate bovine bone graft $\circ \circ$ mixed with blood (Figure 2). Then the vestibular wall was covered by a resorbable collagen membrane. Flap closure was completed using silk interrupted sutures. The sutures were removed 7 days after the surgery. After 90 days of healing, implants were loaded.

\section{Clinical Variables}

The extracted clinical variables were: assessment of prosthetic and implant mobility, presence of inflammation or suppuration. As for implant failure: the presence of mobility and either spontaneous or stimulated pain. Implant survival rate was accordingly calculated [14].

\section{Radiographic assessments}

The CT scans were preoperatively (preop) and at 3-year after prosthetic loading (3yrs). Preoperative and postoperative CT scans were superimposed according to Crespi and co-workers (Figure 3) [15]. Then superimposed data have been saved as a file with DICOM extension (Digital Imaging and COmmunications in Medicine) [16]. Once data had been 
processed, volumes (V) were measured as per Sbordone [17] within a standardized Volume Of Interest (VOI) contained within the following boundaries: $10 \mathrm{~mm}$ mesially and $10 \mathrm{~mm}$ distally, $10 \mathrm{~mm}$ buccally and $10 \mathrm{~mm}$ palatally to the center of implant shoulder.

In CT sections the following variables were assessed Bone Volume (BV) before sinus surgery (preop), 3 years after surgery (3yrs) and their difference $(\Delta \mathrm{BV}$ preop $\rightarrow 3 y r s$, from eq.1) or fractional gain (in percentage, from eq.2):

$\Delta \mathrm{BV}=\mathrm{BV}_{3 y r s}-\mathrm{BV}_{\text {preop }} \quad($ eq.1)

$\% \Delta \mathrm{BV}=100 \cdot \frac{\mathrm{BV}_{3 y r s}-\mathrm{BV}_{\text {preop }}}{\mathrm{BV}_{\text {preop }}} \quad($ eq. 2$)$

Marginal bone level (MBL) was evaluated on radiographic cross-sectional images 3 years after surgery. MBL was the distance between the fixture-abutment interface and the most apical point of the bone-to-implant contact. Dental implants were inserted, as recommended, at crestal bone level so MBL was assumed to be close to zero at baseline (just after surgery), so marginal bone loss $(\triangle \mathrm{MBL})$, which could be obtained from equation 3 , was approximated to the 3year marginal bone level. Changes at the mesial and distal $\triangle \mathrm{MBLs}$ were averaged.

$\Delta \mathrm{MBL}=\mathrm{MBL}_{3 y r s}-\mathrm{MBL}_{\text {baseline }} \cong \mathrm{MBL}_{3 y r s} \quad($ eq.3)

\section{Statistical analysis}

Statistical analyses were performed using a statistical tool package $\|$. The Shapiro-Wilk test did not confirm the normal distribution of the outcomes for all the subgroups investigated (Table 1). For a more conservative analysis of pair-wise comparisons, significant differences between times (matched data) were assessed by the Wilcoxon signed-rank test, whereas significant differences between groups (independent data) were identified by Wilcoxon rank-sum test. The level of statistical significance was set at 0.05 .

\section{RESULTS}

Fifty-six patients were originally included in the study (29 in the displacement group and 27 in the filling group). Out of 56 included implants, two (both belonging to the filling group) early failed. Following exclusion criteria finally, 43 patients were selected for further analyses (18 males and 25 females) with a mean age of $56.3 \pm 9.0$ years and with a total of 43 implants enrolled.

\section{Surgical and Prosthetic Findings}

At the 3-year survey, the resulting implant survival rate was $100 \%$ for the displacement group; whereas for filling group, 3-year survival rate was 92.6\%(CI95\%: from $82.7 \%$ to 100\%). In the displacement group, one out of 23 sinus lift 
procedures resulted in perforation of the membrane at the moment of the surgery. A single event of postoperative nasal bleeding was registered (in a male patient), and no associated pain or mobility of dental implant was recorded. Few episodes of minor swelling of the mucosa were reported during the first days of healing for both groups, but neither flap dehiscence, nor mucositis or suppuration was observed. For both groups, the implants were provisionally loaded within 90 days after placement. In the displacement group, the final ceramic restorations were delivered within 17 weeks after surgery, whilst in the filling group, final restoration was placed into function within 36 weeks from augmentation surgery.

\section{Radiological evaluation}

Radiographic volumes of the bone beneath the sinus were measured in the VOI before and three years after sinus augmentation (Table 1 and Figure 4). Significant differences were recorded between times showing gains in the bone volume of $0.36 \pm 0.17 \mathrm{cc}$ and $0.71 \pm 0.31 \mathrm{cc}$, for displacement and filling group, respectively. An increase in bone resulted by measuring volume of the alveolar bone beneath the displaced sinus in the region inside the VOI from preoperative time $(1.17 \pm 0.34 \mathrm{cc})$ to 3 -year follow-up $(1.53 \pm 0.39 \mathrm{cc})$ with a $\mathrm{p}$-value of 0.0017 ; again the bone beneath the maxillary filled sinus within the VOI increased from $1.24 \pm 0.41 \mathrm{cc}$ to $1.94 \pm 0.68 \mathrm{cc}$ with a significant difference (p-value $<0.0001)$. In terms of percentage, the increase of the bone volume after the backward distraction of the antero-lateral portion of the sinus floor $(\Delta \%)$, was $34 \pm 21 \%$. The sinus lifting and grafting with particulate bovine material allowed a bone gain of $57 \pm 13 \%$.

Marginal bone loss around fixtures at 3 years after implant placement was $1.47 \pm 0.38 \mathrm{~mm}$ and $1.30 \pm 0.58 \mathrm{~mm}$, for the displacement and filling group respectively (Table 1). No significance between the two groups was registered except for the distal aspect of the implant, in which bone loss was $1.66 \pm 0.72 \mathrm{~mm}$ for displacement and $1.25 \pm 0.78 \mathrm{~mm}$ for filling (with p-value $=0.0497)$.

\section{DISCUSSION}

Even though the use of tilted dental implants and short/ultrashort implants beneath the sinus seem to guarantee an adequate clinical performance, when the bone height is judged insufficient for the above-mentioned rehabilitation strategies, augmentation procedures are required [18]. The osteotomy technique seemed to be adequate for elevating the Schneiderian membrane when the residual bone height is at least $5 \mathrm{~mm}$. When bone loss is more accentuated, a lateral antrostomy is generally recommended for placing fixtures with adequate length [19]. However, bone augmentation in horizontal and vertical direction could be the solution when the pristine bone was not adequate for standard implants with or without the use of bone substitute materials. 
The rationale of the study was to report the middle-term effectiveness of the backward displacement of the anterior wall of the maxillary sinus by using electro-magnetic devices and osteotomes in patients with bone thickness beneath sinus close to $3 \mathrm{~mm}$ (that is a class D maxillary sinus) [3].

After the creation of a "greenstick" fracture malleting the pristine maxillary sinus floor, the clinician compressed the spongy bone within the osteotomy site up and backward to increase the amount of available bone for adequate implant placement. The radiographic analysis reported an increase of the available bone with a mean bone gain of $0.36 \mathrm{cc}$, being the bone supporting the dental implant at the distal aspect stable for at least three years. This datum was compared with outcomes of a sinus augmentation procedure, that is, the sinus lifting with lateral access and grafting with particulate bovine graft, which showed, 3-year after augmentation surgery, a mean bone gain of $0.71 \mathrm{cc}$. It appeared that the increase in the bone volume of the test surgery was about half that of the standard sinus lift and grafting technique. However, this was because, when a bone lid technique was applied, the clinician preferred to increase as much as possible the space between the sinus floor and the elevated Schneiderian membrane. The backward displacement of the anterior sinus wall seemed to be a less-demanding procedure, and however, it allowed a sufficient bone volume to properly support a dental implant placed in an edentulous bicuspid area.

The only study describing a volume gain in the atrophic maxillas underwent sinus lift augmentation with a bovine bone substitute material was the case series of Scarano in which he reported a volume ranging from 1.40 to $2.81 \mathrm{cc}$ at 6 month follow-up [20]; the values were in line with the present volumetric outcomes.

To the best of our knowledge, there seemed to be no data regarding the results of three-dimensional volume change measurements of techniques similar to that described in this paper; however, when healing of a cortico-cancellous block bone grafted into the maxillary sinus was investigated a re-pneumatization phenomenon had been reported between the first and the second year. The graft resorption was close to $50 \%$ with a mean volume of the grafted bone of $0.66 \mathrm{cc}$ which authors had seen could be reabsorbed, moreover, their procedure was a more demanding surgery than displacement procedure that was described in the present paper [21].

While some studies attested, after maxillary sinus lift with a membrane elevation procedure, a mean linear bone gain in the range of 5.67-10.9mm [22-24], several other authors experienced, with osteotome technique, a bone gain lower than abovementioned and ranging from $1.8 \mathrm{~mm}$ to $3.94 \mathrm{~mm}$ [25-29]. Even if the technique did not appear to be similar to those described in the present work, outcomes confirmed that sinus lift carried out with a lateral window approach led to a higher increase of the bone volume than that obtained for osteotome-mediated transcrestal sinus lift approach.

Survival rate after maxillary sinus augmentation with a lateral window approach was 92.6\%; it was lower than that of the displacement group (100\%) and very similar to that registered for the osteotome sinus floor elevation (OSFE), which registered a rate between 2 and 3 years in the range of 87.5-98.7\% [22-24,30]. 
Peri-implant marginal bone losses after 3 -year functional loading were $1.47 \mathrm{~mm}$ and $1.30 \mathrm{~mm}$ for the displacement and filling group, respectively and they appeared to be in line with the survival data registered by other authors. Radiological outcomes after OSFE suggested a MBL in the range of $0.8-1.3 \mathrm{~mm}[28,29,31]$, whereas the peri-implant MBL after maxillary sinus augmentation with a lateral window approach ranged between $1.0 \mathrm{~mm}$ and $1.8 \mathrm{~mm}[23,32]$. From the present point of view, a clinician, who want to plan an implant-supported fixed rehabilitation in the maxillary first/second premolar edentulous site close to the anterior wall of the sinus floor, should consider backward displacement of the anterior wall giving the same results as traditional surgery, that is, sinus augmentation with a lateral window approach. Nevertheless, no xenogeneic bone material was used in the augmentation procedure, a great amount of bone gain was obtained after 3-year, suggesting that the technique was highly reliable and successful.

Given the nature of the study, it should be noted that the presence of data regarding bone volume remodeling remains one of the most important criteria. On the other hand, the strength of the present study was the single brand and type of dental implant and the uniformity of surgical performances.

\section{CONCLUSIONS}

However, clinical and radiographic outcomes related to implants presented in this study showed an effective bone gain around dental implants at a 3-year survey for sinus augmented both by backward displacement of the anterior wall and by sinus lifted with a lateral window approach and grafted with particulate bone. The displacement procedure seemed to have a higher success rate and lead to a slightly higher marginal bone loss at the distal aspect than the conventional technique of sinus lift. 


\section{DECLARATIONS}

\section{Ethics Approval And Consent To Participate}

All procedures performed in studies involving human participants were in accordance with the ethical standards of the institutional and/or national research committee and with the 1964 Helsinki declaration and its later amendments or comparable ethical standards. Approval for this retrospective analysis was obtained from the Ethical Committee of the University of Pisa, Pisa, Italy (Ethical Approval Form 2626/2008 Protocol Number 58183)

\section{Consent For Publication}

Written informed consent was obtained from all subjects included in the present retrospective analysis.

\section{Availability Of Data And Materials}

The file were encrypted in a dedicated database (.mat). However, patients did not give us the consent to send raw data.

\section{Competing Interests}

The authors declare that they have no competing interests. All the authors and the authors' institutions have neither financial nor personal relationships with other people or organizations who might inappropriately influence their actions.

\section{Funding}

The work was supported by the Study Center for Regenerative Research, University "G. Marconi”, Rome Italy

\section{Authors' Contributions}

The authors met all criteria requested for authorship. A short description of each authors' contribution is given below. 


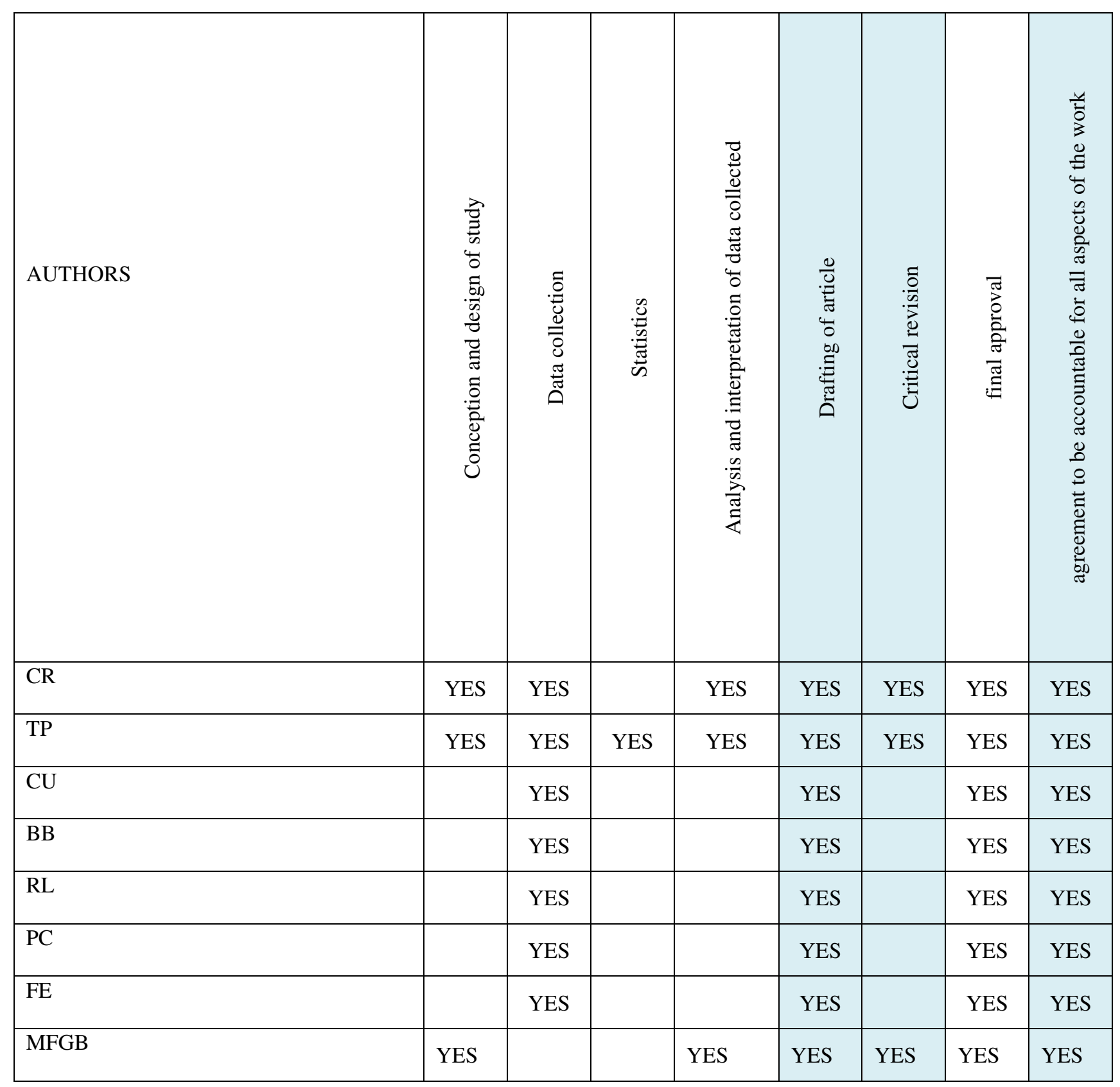

\section{Acknowledgements}

Not applicable

\section{Authors' Information (Optional)}

Not applicable

\section{Footnotes}

$\dagger \dagger$ optocaine $20 \mathrm{mg} / \mathrm{ml}$ with adrenaline 1:80.000, Molteni Dental, Scandicci, FI, Italy

†† Magnetic Mallet, Osseotouch Total Control, contact@osseotouch.com, Turbigo, Milano, Italy 
$\S \S$ Outlink, Sweden Martina: rough titanium plasma spray surface, $0.8 \mathrm{~mm}$ with a machined collar and progressive thread design

** Gingistat, Acteon Pharma, Bordeaux, France

${ }^{\circ}$ Bio-Oss $^{\circledR}$, 0.5-1 $\mu \mathrm{m}$ particle-size, Geistlich Biomaterials Italia srl, Thiene, VI, Italy

|| Statistics Toolbox, MatLab 7.11; The MathWorks 


\section{REFERENCES}

[1] Khoury F. Augmentation of the sinus floor with mandibular bone block and simultaneous implantation: A 6-year clinical investigation. Int J Oral Maxillofac Implants. 1998;14:557-64

[2] Boyne PJ, James RA. Grafting of the maxillary sinus floor with autogenous marrow and bone. J Oral Surg. $1980 ; 38: 613-6$

[3] Jensen OT, Shulman LB, Block MS et al. Report of the sinus consensus conference of 1996. Int J Oral Maxillofac Implants. 1998;13(Suppl):11-45

[4] Pjetursson BE, Tan WC, Zwahlen M et al. A systematic review of the success of sinus floor elevation and survival of implants inserted in combination with sinus floor elevation. Part I: lateral approach. J Clin Periodontol. 2008;35(Suppl 8):216-240

[5] Tatum H Jr. Maxillary and sinus implant reconstructions. Dent Clin North Am. 1986;30:207-29

[6] Summers RB. A new concept in maxillary implant surgery: the osteotome technique. Compendium. 1994;15:152-8

[7] Summers RB. The osteotome technique: part 3 - Less invasive methods of elevating the sinus floor. Compendium. 1994;15:698-704

[8] Deporter D, Todescan R, Caudry S. Simplifying management of the posterior maxilla using short, porous surfaced dental implants and simultaneous indirect sinus elevation. Int J Periodontics Restorative Dent. 2000;20:476-85

[9] Bruschi GB, Crespi R, Capparè P et al. Transcrestal Sinus Floor Elevation: A Retrospective Study of 46 Patients up to 16 Years. Clin Implant Dent Rel Res. 2012;14:759-67

[10] Esposito M, Grusovin MG, Rees J et al. Effectiveness of sinus lift procedures for dental implant rehabilitation: A Cochrane systematic review. Eur J Oral Implantol. 2010;3:7-26 
[11] Langer B, Langer L. Overlapped flap: a surgical modification for implant fixture installation. Int J Periodont Rest Dent. 1990;10:208-15

[12] Crespi R, Capparè P, Gherlone E. Electrical Mallet Provides Essential Advantages in Maxillary Bone Condensing. A Prospective Clinical Study. Clin Implant Dent Relat Res. 2013;15:874-82

[13] Crespi R, Capparè P, Gherlone E. Sinus Floor Elevation By Osteotome. Hand Mallet Versus Electrical Mallet: A Prospective Clinical Study. Int J Oral Maxillofac Implants. 2012;27:1144-50

[14] Romeo E, Lops D, Margutti E et al. Long-term survival and success of oral implants in the treatment of full and partial arches: a 7-year prospective study with the ITI dental implant system. Int J Oral Maxillofac Implants. 2004; $19: 247-59$

[15] Crespi R, Fabris GBM, Crespi G et al. Effects of different loading protocols on the bone remodeling volume of immediate maxillary single implants: A 2- to 3-year follow-up. Int J Oral Maxillofac Implants. 2019;34:953-62

[16] Sbordone C, Toti P, Martuscelli R et al. Evaluation of volumetric dimensional changes in posterior extraction sites with and without ARP using a novel imaging device. Clin Implant Dent Relat Res. 2017;19:1044-53

[17] Sbordone C, Toti P, Martuscelli R et al. Retrospective volume analysis of bone remodeling after tooth extraction with and without deproteinized bovine bone mineral insertion. Clin Oral Implants Res. 2016;27:1152-9

[18] Lombardo G, Pighi J, Marincola M et al. Cumulative Success Rate of Short and Ultrashort Implants Supporting Single Crowns in the Posterior Maxilla: A 3-Year Retrospective Study. Int J Dent. 2017;2017:8434281

[19] Pal US, Sharma NK, Singh RK et al. Direct vs. indirect sinus lift procedure: A comparison. Natl J Maxillofac Surg. $2012 ; 3: 31-7$

[20] Scarano A. Maxillary Sinus Augmentation with Decellularized Bovine Compact Particles: A Radiological, Clinical, and Histologic Report of 4 Cases. Biomed Res Int. 2017;2017:2594670 
[21] Sbordone C, Toti P, Guidetti F et al. Volume changes of autogenous bone after sinus lifting and grafting procedures: a 6-year computerized tomographic follow-up. J Craniomaxillofac Surg. 2013;41:235-41

[22] Borges FL, Dias RO, Piattelli A et al. Simultaneous sinus membrane elevation and dental implant placement without bone graft: a 6-month follow-up study. J Periodontol. 2011;82:403-12

[23] Cricchio G, Sennerby L, Lundgren S. Sinus bone formation and implant survival after sinus membrane elevation and implant placement: a 1- to 6-year follow-up study. Clin Oral Implants Res. 2011;22:1200-12

[24] Riben C, Thor A. Follow-Up of the Sinus Membrane Elevation Technique for Maxillary Sinus Implants without the Use of Graft Material. Clin Implant Dent Relat Res. 2016;18:895-905

[25] Leblebicioglu B, Ersanli S, Karabuda C et al. Radiographic evaluation of dental implants placed using an osteotome technique. J Periodontol. 2005;76:385-90

[26] Lai HC, Zhuang LF, Lv XF et al Osteotome sinus floor elevation with or without grafting: a preliminary clinical trial. Clin Oral Implants Res. 2010;21:520-6

[27] Fornell J, Johansson LA, Bolin A et al. Flapless, CBCT-guided osteotome sinus floor elevation with simultaneous implant installation. I: radiographic examination and surgical technique. A prospective 1-year follow-up. Clin Oral Implants Res. 2012;23:28-34

[28] Brizuela A, Martín N, Fernández-Gonzalez FJ et al. Osteotome sinus floor elevation without grafting material: Results of a 2-year prospective study. J Clin Exp Dent. 2014;6:e479-84

[29] Nedir R, Nurdin N, Vazquez L et al. Osteotome Sinus Floor Elevation without Grafting: A 10-Year Prospective Study. Clin Implant Dent Relat Res. 2016;18:609-17

[30] Bortoluzzi MC, Manfro R, Fabris V et al. Comparative study of immediately inserted dental implants in sinus lift: 24 months of follow-up. Ann Maxillofac Surg. 2014;4:30-3 
[31] Si MS, Zhuang LF, Gu YX et al. Osteotome sinus floor elevation with or without grafting: a 3-year randomized controlled clinical trial. J Clin Periodontol. 2013;40:396-403

[32] Cricchio G, Imburgia M, Sennerby L et al. Immediate loading of implants placed simultaneously with sinus membrane elevation in the posterior atrophic maxilla: a two-year follow-up study on 10 patients. Clin Implant Dent Relat Res. 2014;16:609-17 


\section{LEGENDS TO TABLES}

Table 1. Mean and standard deviation of volume at pre-operative (preop) and at 3-year survey (3yrs) of the alveolar bone, and their difference, $\Delta \mathrm{BV}$ (with $\% \Delta \mathrm{BV}$ that is its percentage) from pre-operative to 3-year survey (preop $\rightarrow 3 \mathrm{yrs}$ ) in the Volume Of Interest (VOI). Marginal bone loss from pre-operative to 3-year survey (preop $\rightarrow$ 3yrs). Normal distribution test: ${ }^{\wedge}$ (Shapiro-Wilk test); statistical comparisons: $*$ Wilcoxon signed-rank test assessing changes in time from pre-operative to 3 -year follow-up; ${ }^{\circ}$ Wilcoxon rank-sum test assessing changes between groups. 


\section{CAPTIONS TO FIGURES}

Figure 1. Augmented sinus by backward displacement technique of the anterior wall: (A-B) Procedure scheme for the surgical treatment; (C) Clinical photograph showing the edentulous ridge of the maxilla before surgery; (D) Exposure of the crest with the partial-thickness flap with placed implant; (E) Clinical view of surgical procedure with final sutures of gingival margins; (F) Clinical photographs three months after surgical procedure.

Figure 2. Augmented sinus by lifting with a lateral window approach and grafting with particulate bone substitute: (A) Exposure of the crest with full-thickness flap with osteotomies; (B) Dental implants placed sinus lifted and grafted with xenogeneic bone substitute material; (C) Surgical site covered by reabsorbable membrane; (D) Clinical view of surgical procedure with final sutures of gingival margins;

Figure 3. Fontal and lateral views of an augmented sinus by backward displacement technique of the anterior wall: A) Preoperative; B) Postoperative; C) Fused files. Fontal and lateral views of an augmented sinus by lifting with a lateral window approach and grafting with particulate bone substitute: D) Preoperative; E) Postoperative; F) Fused files.

Figure 4. Box and wishers plot (with scatter data) of the volume at pre-operative $\left(\mathrm{BV}_{\text {preop }}\right)$ and at 3 -year survey $\left(\mathrm{BV}_{3 \mathrm{yrs}}\right)$ of alveolar bone beneath the sunus, and their difference, $\Delta \mathrm{BV}$ (with its percentage $\% \Delta \mathrm{BV}$ ) from pre-operative to 3-year survey (preop $\rightarrow 3 \mathrm{yrs}$ ) in the Volume Of Interest (VOI). Results for pairwise statistical comparisons: Wilcoxon signedrank test assessing changes in time from pre-operative and 3-year follow-up (*); Wilcoxon rank-sum test assessing changes between groups $\left(^{\circ}\right)$. 


\section{Figures}
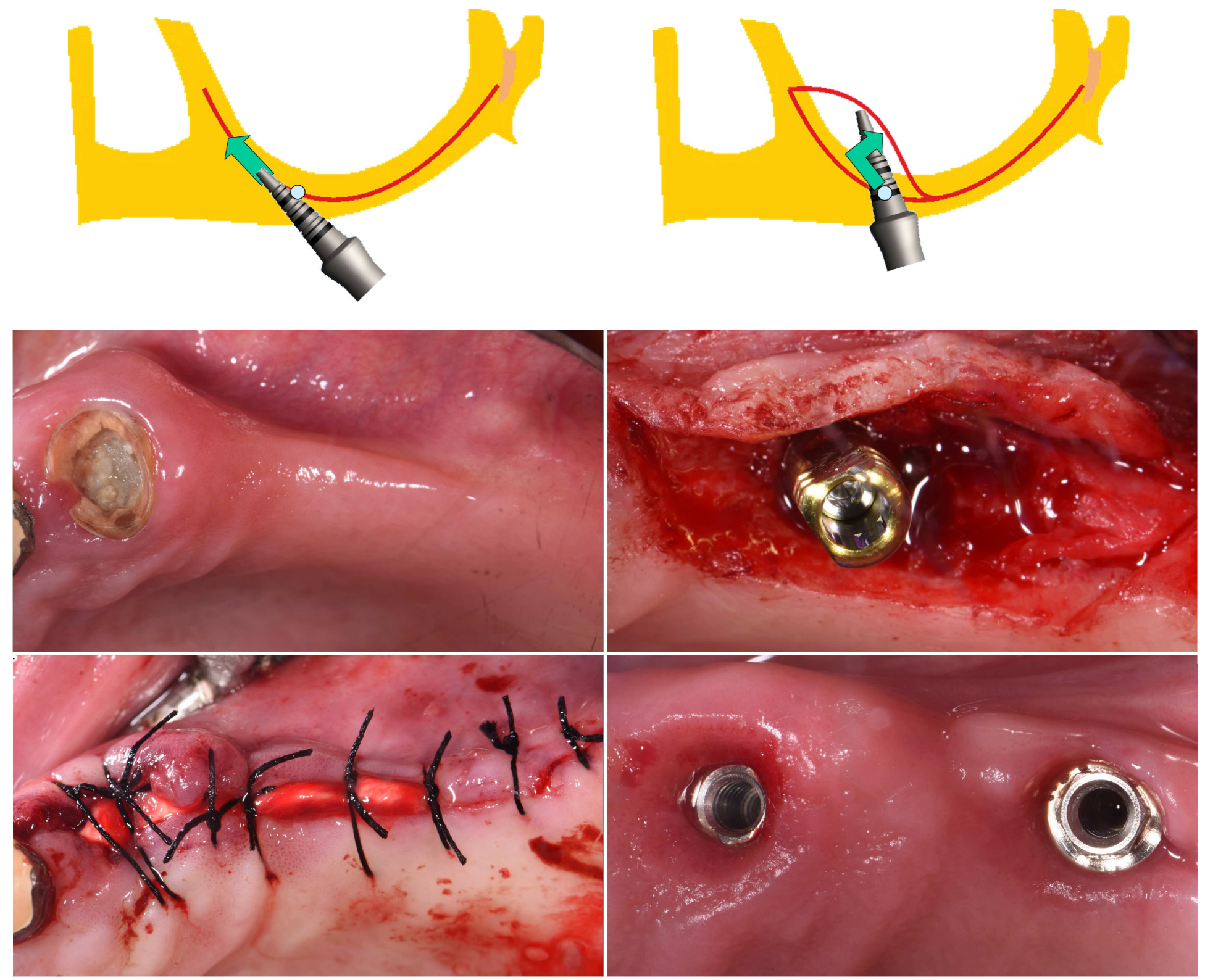

\section{Figure 1}

Augmented sinus by backward displacement technique of the anterior wall: (A-B) Procedure scheme for the surgical treatment; (C) Clinical photograph showing the edentulous ridge of the maxilla before surgery; (D) Exposure of the crest with the partial-thickness flap with placed implant; (E) Clinical view of surgical procedure with final sutures of gingival margins; (F) Clinical photographs three months after surgical procedure. 


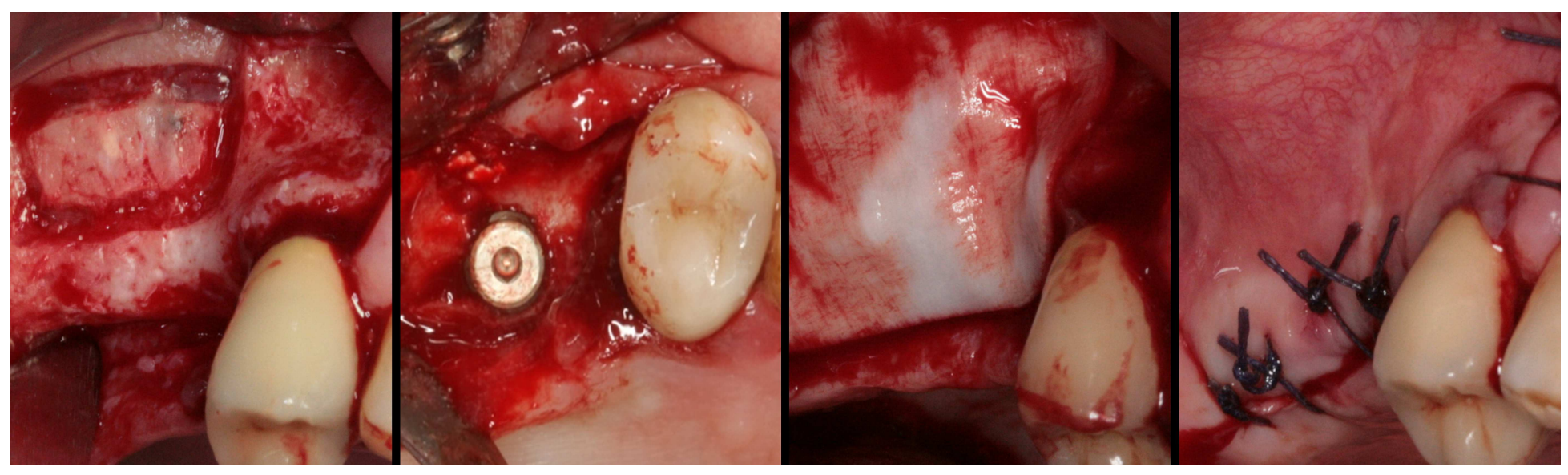

\section{Figure 2}

Augmented sinus by lifting with a lateral window approach and grafting with particulate bone substitute: (A) Exposure of the crest with full-thickness flap with osteotomies; (B) Dental implants placed sinus lifted and grafted with xenogeneic bone substitute material; (C) Surgical site covered by reabsorbable membrane; (D) Clinical view of surgical procedure with final sutures of gingival margins; 


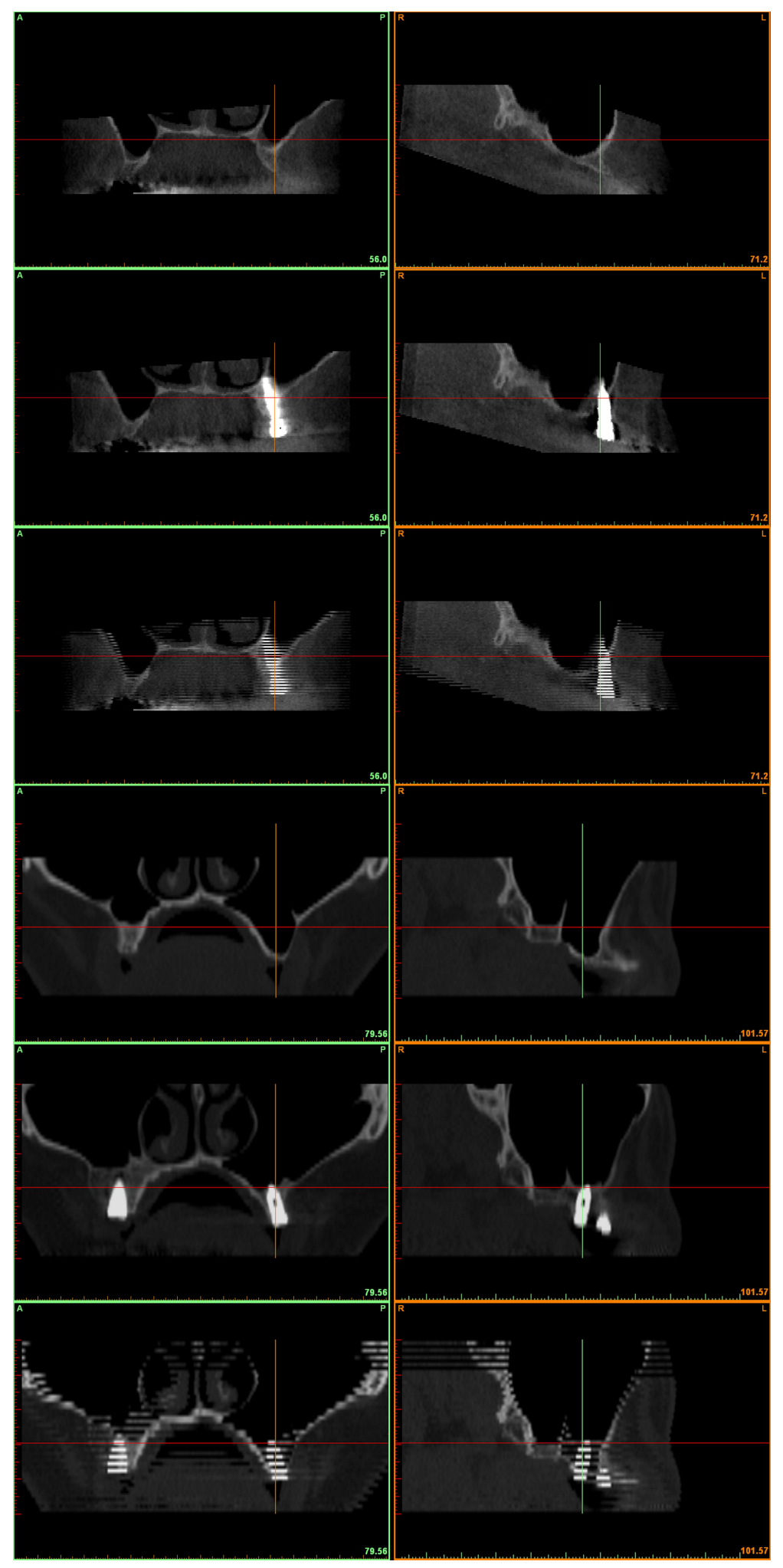

\section{Figure 3}

Fontal and lateral views of an augmented sinus by backward displacement technique of the anterior wall: A) Preoperative; B) Postoperative; C) Fused files. Fontal and lateral views of an augmented sinus by lifting with a lateral window approach and grafting with particulate bone substitute: D) Preoperative; E) Postoperative; F) Fused files. 


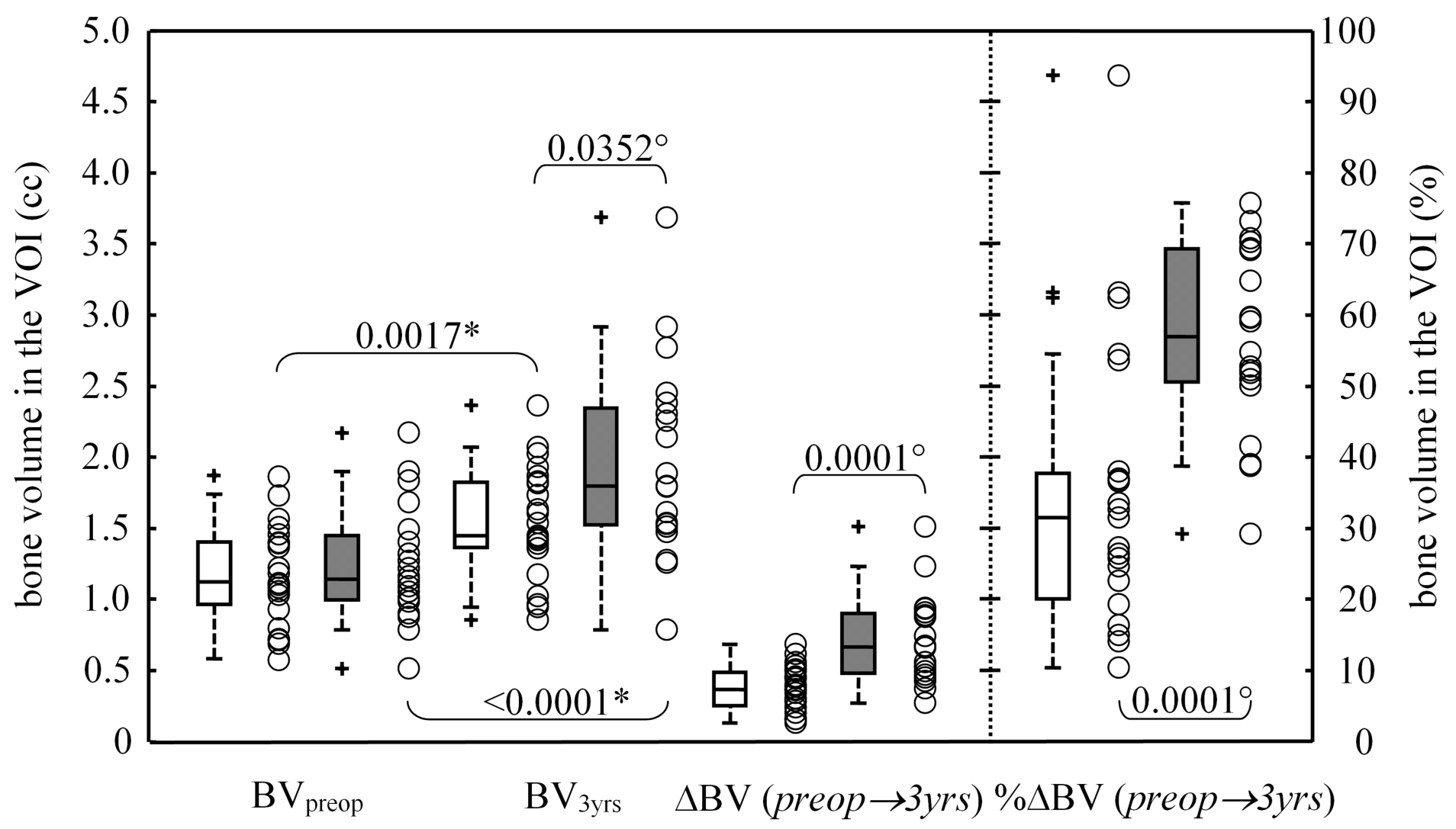

Figure 4

Box and wishers plot (with scatter data) of the volume at pre-operative (BVpreop) and at 3-year survey (BV3yrs) of alveolar bone beneath the sunus, and their difference, पBV (with its percentage \% $\mathrm{BV}$ ) from pre-operative to 3-year survey (preop囚3yrs) in the Volume Of Interest (VOI). Results for pairwise statistical comparisons: Wilcoxon signed-rank test assessing changes in time from pre-operative and 3-year followup $\left({ }^{\star}\right)$; Wilcoxon rank-sum test assessing changes between groups $\left(^{\circ}\right)$. 